\title{
Quantum metrology in a non-Markovian quantum evolution
}

\author{
Nicolás Mirkin $\odot,{ }^{*}$ Martín Larocca, and Diego Wisniacki \\ Departamento de Física “J. J. Giambiagi” and IFIBA, FCEyN, Universidad de Buenos Aires, 1428 Buenos Aires, Argentina
}

(Received 12 December 2019; revised 1 July 2020; accepted 7 August 2020; published 24 August 2020)

\begin{abstract}
The problem of quantum metrology in the context of a particular non-Markovian quantum evolution is explored. We study the dynamics of the quantum Fisher information (QFI) of a composite quantum probe coupled to a Lorentzian environment, for a full variety of different classes of parameters. We are able to find the best metrological state, which is not maximally entangled but is the one which evolves the most rapidly. This is shown by demonstrating a connection between QFI and different quantum speed limits. At the same time, by optimizing a control field acting on the probes, we show how the total information flow is actively manipulated by the control so as to enhance the parameter estimation at a given final evolution time. Finally, under this controlled scenario, a sharp interplay between the dynamics of QFI, non-Markovianity, and entanglement is revealed within different control schemes.
\end{abstract}

DOI: 10.1103/PhysRevA.102.022618

\section{INTRODUCTION}

One of the most developed areas within the advent of quantum information technologies during the last two decades of quantum revolution has undoubtedly been quantum metrology. This field is responsible for the development of highresolution and highly sensitive measurements of physical parameters, which is a central task for the prosperous evolution of technology [1,2]. In this context, the question of whether the powerful resources of nature that have been revealed by quantum mechanics can be exploited to improve the precision in the estimation of unknown parameters associated with a quantum system has been widely studied in the literature [3-8].

Since any realistic quantum system interacts and exchanges information with an environment, the main challenge resides in tackling the problem of quantum metrology within the presence of decoherence and non-Markovianity (NM) [9-22]. Because both phenomena are related to the loss and gain of information, respectively, the question of how the dynamics of estimation is affected by the presence of both decoherence and NM is of paramount interest and worthy of study. In that sense, as memory effects-usually associated with non-Markovian quantum processes-allow us to recover information from the environment that otherwise would be lost $[23,24]$, this has opened a new door for applications in quantum metrology.

A natural question then arises: Is it possible to exploit some particular feature of the environment in order to enhance the precision of estimation of different classes of parameters? For instance, it has recently been shown that NM can be actively manipulated to generate a controlled degree of entanglement between two noninteracting subsystems coupled to the same non-Markovian reservoir $[25,26]$ and, also, that dissipation

\footnotetext{
*mirkin@df.uba.ar
}

can be engineered to be a fully fledged resource for universal quantum computation [27]. So the possibility of engineering some particular feature of the environment to improve the process of estimating different classes of parameters certainly seems plausible [28].

In this work, we analyze a quantum metrology scenario within a particular non-Markovian quantum evolution. By using two noninteracting subsystems, we focus on the capability of this composite quantum system to act as a probe and to extract relevant information on parameters characterizing a common structured environment to which they are coupled. Seeking universality, we also focus on the process of estimation of parameters characterizing the interaction and the quantum probe itself. By exploring the role of initial entanglement within the composite probe in all the cases just mentioned, we find that the best metrological candidates are not the maximally entangled states but the ones that evolve the most rapidly. This is shown using the quantum speed limit (QSL), a tool that characterizes the minimum time a quantum system needs in order to travel a predetermined distance in Hilbert space [29-33]. Thus, while demonstrating that the speed of evolution and the accuracy of the estimation are deeply connected in all the metrological situations covered, we also show that entanglement is not decisive to accelerate a non-Markovian evolution or truly useful for improving the estimation of the parameter of interest.

Another important key point of our work resides in the implementation of optimal control tools, allowing us to achieve a controlled degree of precision in the estimation of the unknown parameter at a given final evolution time. Therefore, after identifying the best metrological candidates, we show how by optimizing a control field over the composite probe, the total information flowing throughout the evolution can be actively accommodated by the control so as to maximize the precision of the estimation at this given final evolution time. Finally, by exploring different control schemes, we reveal a direct dynamical relation between the information flows 
regarding the precision of estimation, NM, and entanglement in some specific circumstances.

The paper is organized as follows. Section II reviews the main concepts of quantum metrology theory. Section III summarizes the concept of the QSL for nonunitary dynamics by presenting two of the most well-known bounds that have been previously derived in the literature. Section IV analyzes the main features characterizing NM together with a measure able to quantify it. Section $V$ provides the physical model on which we based our study and in Sec. VI the main results obtained with this model are presented. Finally, Sec. VII concludes with some final remarks.

\section{QUANTUM METROLOGY THEORY}

In this section, we provide a brief summary of the most relevant features of quantum metrology theory. The purpose of quantum metrology is to deal with estimation processes within quantum systems, pursuing the best precision that is physically allowed [1,2]. For instance, let us consider a situation in which the quantum evolution of a certain system is known except for a certain parameter $\tilde{\lambda}$. This $\tilde{\lambda}$ may be estimated from the knowledge of the initial and final states of a given probe that undergoes the process of interest. The metrological procedure is usually the following: a quantum probe is first initialized in a particular input state and, as it evolves, is transformed into a mixed state encoding information on the unknown parameter $\tilde{\lambda}$. After the evolution, a suitable measurement must be done over the probe so as to extract information about it. Finally, each experimental result should be associated with some estimator of the parameter of interest. In this context, any measurement of a certain observable $X$ is associated with an outcome $x$ that occurs with a conditional probability distribution $p_{X}(x \mid \tilde{\lambda})$, which is defined by $[17,34,35]$

$$
p_{X}(x \mid \tilde{\lambda})=\operatorname{Tr}\left(P_{x} \rho_{\tilde{\lambda}}\right) .
$$

Above, $\rho_{\tilde{\lambda}}$ refers to the quantum state of the probe and $P_{x}$ to positive operator-valued measures, satisfying the relation $\sum_{x} P_{x}=\mathbb{1}$, usually known in the literature as POVMs. Therefore, in order to estimate the value of our unknown parameter $\tilde{\lambda}$ from the outcome measurements, an estimator is needed. This estimator must be a function of the measurement outcomes, i.e., $\hat{\tilde{\lambda}}=\hat{\tilde{\lambda}}\left(x_{1}, x_{2}, \ldots\right)$, and also should satisfy certain properties, such as being unbiased,

$$
E[\hat{\tilde{\lambda}}-\tilde{\lambda}]=\prod_{i} \sum_{x_{i}} \hat{\tilde{\lambda}}\left(x_{1}, \ldots, x_{n}\right)-\tilde{\lambda}=0 \quad \forall \tilde{\lambda},
$$

where $E[\cdot]$ corresponds to the mean with respect to the $n$ outcomes $x_{i}$ and $\tilde{\lambda}$ denotes the true value of the parameter. Moreover, it is also important to require a small variance for the estimator, i.e., $\operatorname{Var}(\tilde{\lambda}, \hat{\tilde{\lambda}})=E\left[\hat{\tilde{\lambda}}^{2}\right]-E[\tilde{\lambda}]^{2}$, considering that this quantity measures the overall precision of the inference process [35]. With regard to this quantity, it is well known that a lower bound for the variance of any estimator is imposed by the Cramér-Rao theorem [36,37],

$$
\operatorname{Var}(\tilde{\lambda}, \hat{\tilde{\lambda}}) \geqslant \frac{1}{M G_{\tilde{\lambda}}},
$$

where $M$ denotes the number of independent measurements and $G_{\tilde{\lambda}}$ is known as the Fisher information (FI) and is defined by

$$
G_{\tilde{\lambda}}=\sum_{x} \frac{\left[\partial_{\tilde{\lambda}} p_{X}(x \mid \tilde{\lambda})\right]^{2}}{p_{X}(x \mid \tilde{\lambda})} .
$$

The challenge is then to choose the best estimator so as to achieve an optimal inference and saturate the CramérRao bound. The fact that different observables will lead to different probability distributions is intuitive, which means that each one will be associated with a particular FI and hence to different precisions for the estimation of the unknown parameter $\tilde{\lambda}$ [8]. The ultimate bound is traditionally obtained upon maximizing the FI over the set of all possible POVMs. The best measurement that provides the maximum precision is quantified with what is called the quantum Fisher information (QFI), which is given by

$$
\mathcal{F}_{\tilde{\lambda}}=\sum_{n} \frac{\left(\partial_{\tilde{\lambda}} \omega_{n}\right)^{2}}{\omega_{n}}+2 \sum_{n \neq m} \frac{\left(\omega_{n}-\omega_{m}\right)^{2}}{\omega_{n}+\omega_{m}}\left\|\left\langle\psi_{n} \mid \partial_{\tilde{\lambda}} \psi_{m}\right\rangle\right\|^{2},
$$

where $\left\{\omega_{n}\right\}$ are the eigenvalues of the reduced density matrix of the probe and $\left\{\left|\psi_{n}\right\rangle\right\}$ its eigenvectors. In this way, the QFI is lower bounded by the FI, i.e., $G_{\tilde{\lambda}} \leqslant \mathcal{F}_{\tilde{\lambda}}$. Note that $\tilde{\lambda}$ can be any parameter characterizing either the probe, the interaction, or even the environment. Let us, finally, stress that since $\mathcal{F}_{\tilde{\lambda}}(t)$ is truly a dynamical quantity, in this work we will sometimes work with the total QFI throughout a particular given evolution, i.e.,

$$
\mathcal{F}_{\tilde{\lambda}}^{(\text {tot })}=\int_{0}^{T} \mathcal{F}_{\tilde{\lambda}}\left(t^{\prime}\right) d t^{\prime},
$$

with $T$ referring to some fixed evolution time. This total QFI gives us an average of the information that could be obtained throughout the whole dynamics. A higher $\mathcal{F}_{\tilde{\lambda}}^{(\text {tot })}$ implies a greater possibility of achieving a better degree of precision at some particular time and thus it is an indicator of how well an initial state could potentially act as a metrological state. Of course, we emphasize that what is really important in the end is the QFI at a fixed evolution time [i.e., $\mathcal{F}_{\tilde{\lambda}}(T)$ ] and not throughout the whole evolution, but we will show with optimal control tools that these two quantities are strongly related. In fact, by addressing the system with an external control field, the optimal QFI achieved for a specific final evolution time $T$ will coincide with the global maximum reached at an arbitrary time throughout the whole noncontrolled evolution (i.e., $\left.\mathcal{F}_{\tilde{\lambda}}^{\text {(opt) }}(T)=\max \left(\mathcal{F}_{\tilde{\lambda}}(t)\right) \forall t \in[0, T]\right)$.

\section{QUANTUM SPEED LIMITS FOR OPEN QUANTUM EVOLUTIONS}

In this section, we review two of the most well-known QSLs derived in the literature for nonunitary quantum evolutions. The QSL time $\tau$ is defined as the minimal time a quantum system needs in order to evolve from an initial to a final state, separated by a given predetermined distance [29-33]. The first approach to the correct formulation of the QSL we present is based on the definition of the Bures fidelity between 
an initial and a final state, i.e. [31,32],

$$
F_{B}\left(\rho_{0}, \rho_{t}\right)=\operatorname{Tr}\left(\sqrt{\sqrt{\rho_{0}} \rho_{t} \sqrt{\rho_{0}}}\right) .
$$

It can be proven that the tightest lower bound for the actual path length of the evolution is given by the Bures angle,

$$
\mathcal{L}\left(\rho_{0}, \rho_{t}\right) \equiv \arccos \left(F_{B}\left(\rho_{0}, \rho_{t}\right)\right) \leqslant \int_{0}^{t} \sqrt{\frac{\mathcal{F}_{t}\left(t^{\prime}\right)}{4}} d t^{\prime},
$$

where $\mathcal{F}_{t}(t)$ corresponds to the QFI for time estimation and $\mathcal{L}\left(\rho_{0}, \rho_{t}\right)$ to the Bures angle, which is a predetermined distance [i.e., between orthogonal states $\mathcal{L}\left(\rho_{0}, \rho_{t}\right)=\pi / 2$ ]. Therefore, since $\sqrt{\frac{\mathcal{F}_{t}(t)}{4}}$ is commonly regarded as the instantaneous speed of evolution [31], the time that saturates that fixed predetermined distance defines the minimum time of evolution, the QSL, which we call $\tau_{\mathcal{F}}$ :

$$
\mathcal{L}\left(\rho_{0}, \rho_{t}\right)=\int_{0}^{\tau_{\mathcal{F}}} \sqrt{\frac{\mathcal{F}_{t}\left(t^{\prime}\right)}{4}} d t^{\prime} .
$$

In other words, $\tau_{\mathcal{F}}$ reflects the time that the system takes to travel-along the actual evolution path - the same length as the geodesic's length between two predetermined states. Moreover, as already proven in Ref. [32], this expression for the QSL is the only one that sticks close to the essence of the QSL theory [38-40] since it is always possible to find an evolutionary path that, for every time, saturates the bound. This will occur whenever the system evolution equals the geodesic path.

Another very popular approach used in the literature to derive an expression for the QSL is the one proposed by Deffner and Lutz [29], based on the von Neumann trace inequality for Hilbert-Schmidt class operators. The tightest QSL they found can be consistently defined as

$$
\sin ^{2}\left(\mathcal{L}\left(\rho_{0}, \rho_{t}\right)\right)=\int_{0}^{\tau_{\mathrm{op}}}\left\|\dot{\rho}\left(t^{\prime}\right)\right\|_{\mathrm{op}} d t^{\prime},
$$

where $\|A\|_{\text {op }}$ is the operator norm of $A$. Similarly, the time that saturates the distance fixed by the left-hand side of Eq. (10) corresponds to the QSL time and we denote it $\tau_{\mathrm{op}}$. However, as also demonstrated in Ref. [32], with this approach it becomes impossible to find an evolutionary path where Eq. (10) is saturated at all times.

Let us remark that both expressions presented above for the QSL are used in Sec. VI to illustrate how the speed of evolution and the accuracy of the estimation are closely related within our non-Markovian quantum metrology scenario.

\section{NON-MARKOVIANITY MEASURE}

There are many ways to quantify NM, one of the most popular approaches being related to the revivals of distinguishability and originally proposed by Breuer, Laine, and Piilo (BLP) [23]. The distinguishability can be quantified by the derivative of the trace distance, which is defined as $\mathcal{D}\left(\rho_{1}, \rho_{2}\right)=\frac{1}{2}\left\|\rho_{1}-\rho_{2}\right\|$ and where $\|A\|=\operatorname{tr}\left(\sqrt{A^{\dagger} A}\right)$. Under a Markovian regime, quantum states become less and less distinguishable; there is a continuous loss of information to the environment. But on a non-Markovian regime, distinguishability between states can increase and this is equivalent to saying that information is flowing from the environment back to the system. Therefore, BLP state that a quantum map is non-Markovian if there exists at least a pair of initial states $\rho_{1}(0)$ and $\rho_{2}(0)$ such that the distinguishability between them increases during some interval of time, i.e.,

$$
\sigma\left(\rho_{1}(0), \rho_{2}(0), t\right)=\frac{d}{d t} \mathcal{D}\left(\rho_{1}(t), \rho_{2}(t)\right)>0 .
$$

This idea can also be extended to define a measure of the degree of NM in a quantum process via

$$
\mathcal{N}_{\mathrm{BLP}}=\max _{\left\{\rho_{1}(0), \rho_{2}(0)\right\}} \int_{0, \sigma>0}^{T} \sigma \mathbf{t}\left(\rho_{1}(0), \rho_{2}(0), t^{\prime}\right) d t^{\prime},
$$

where $T$ refers to the final evolution time of the process under consideration. In general, Eq. (12) is integrated to $\infty$, but since here we consider control protocols with a certain finite duration, we quantify NM for a restricted time interval.

\section{PHYSICAL MODEL}

The system we use as a platform to study different aspects of quantum metrology within a non-Markovian quantum evolution consists of two noninteracting two-level atoms, acting as a composite probe, coupled to a common zero-temperature bosonic reservoir composed of a set of $M$-harmonic oscillators $[25,41,42]$. The total microscopic Hamiltonian describing the model is given by

$$
\begin{aligned}
H= & H_{S}+H_{E}+H_{\mathrm{int}} \\
= & \sum_{i=1}^{2} \omega_{i}(t) \sigma_{+}^{(i)} \sigma_{-}^{(i)}+\sum_{k=1}^{M} v_{k} b_{k}^{\dagger} b_{k} \\
& +\sum_{i=1}^{2}\left(a_{i} \sigma_{+}^{(i)} \otimes \sum_{k=1}^{M} g_{k} b_{k}+\text { H.c. }\right),
\end{aligned}
$$

where $\sigma_{j}^{(i)}(j=x, y, z)$ correspond to the Pauli matrices of each atom $(i=1,2), \sigma_{ \pm}^{(i)}=\frac{1}{2}\left(\sigma_{x}^{(i)} \pm i \sigma_{y}^{(i)}\right), b_{k}^{\dagger}$ and $b_{k}$ to the creation and annihilation operators, $g_{k}$ is the coupling constant to the $k$ th mode of the bath and $v_{k}$ its frequency, $a_{i}$ is a dimensionless coupling constant measuring the interaction with the reservoir, and, finally, $\omega_{i}(t)$ refers to the energy difference between the ground $|0\rangle$ and the excited $|1\rangle$ state of atom $i$, which we assume to be time dependent and of the form

$$
\omega_{i}(t)=\omega_{0}+\epsilon_{i}(t)
$$

In principle, $\epsilon_{i}(t)$ is an arbitrary driving field over atom $i$, but for simplicity we work under the framework of global addressing, where $\epsilon_{1}(t)=\epsilon_{2}(t)=\epsilon(t)$. We emphasize that other types of control can also be implemented in our model, such as single addressing $\left[\epsilon_{2}(t)=0 \forall t\right]$ and double addressing $\left[\epsilon_{1}(t) \neq \epsilon_{2}(t) \forall t\right]$, rendering similar results [25]. Assuming that initially the environment has no excitations $\left(\left|0_{B}\right\rangle\right)$ and that the dynamics is restricted to one excitation in the $k$ th 
mode, the whole initial state of the total system is

$$
|\psi(0)\rangle=\left(C_{01}|10\rangle+C_{02}|01\rangle\right) \otimes_{k}\left|0_{B}\right\rangle
$$

and therefore its dynamics is given by

$$
\begin{aligned}
|\psi(t)\rangle= & C_{1}(t)|10\rangle\left|0_{B}\right\rangle+C_{2}(t)|01\rangle\left|0_{B}\right\rangle \\
& +\sum_{k} C_{k}(t)|00\rangle\left|k_{B}\right\rangle,
\end{aligned}
$$

$\left|k_{B}\right\rangle$ being the state of the reservoir with only one excitation in the $k$ th mode $\left(\left|k_{B}\right\rangle=b_{k}^{\dagger}\left|0_{B}\right\rangle\right)$. The next step is to take the continuum limit for the environment, by assuming a Lorentzian spectral density of the form

$$
J(v)=\frac{\mathcal{R}^{2}}{\pi a_{t}^{2}} \frac{\lambda}{\left(v-\omega_{0}\right)^{2}+\lambda^{2}},
$$

where $\mathcal{R}$ is the vacuum Rabi frequency, $a_{t}$ an effective coupling constant defined as $a_{t}=\sqrt{a_{1}^{2}+a_{2}^{2}}$, and $\lambda$ the width of the spectral density of the bath. Finally, it is straightforward to follow the procedure put forward in Ref. [25] and derive these two coupled differential equations for $C_{1}(t)$ and $C_{2}(t)$, respectively:

$$
\begin{aligned}
& \ddot{C}_{1}+(\lambda-i \epsilon(t)) \dot{C}_{1}+\left(\frac{a_{1} \mathcal{R}}{a_{t}}\right)^{2} C_{1}+a_{1} a_{2}\left(\frac{\mathcal{R}}{a_{t}}\right)^{2} C_{2}=0 \\
& \ddot{C}_{2}+(\lambda-i \epsilon(t)) \dot{C}_{2}+\left(\frac{a_{2} \mathcal{R}}{a_{t}}\right)^{2} C_{2}+a_{1} a_{2}\left(\frac{\mathcal{R}}{a_{t}}\right)^{2} C_{1}=0
\end{aligned}
$$

The density matrix can be written as $[41,42]$

$$
\rho(t)=\left(\begin{array}{cccc}
0 & 0 & 0 & 0 \\
0 & \left|C_{1}(t)\right|^{2} & C_{1}(t) C_{2}^{*}(t) & 0 \\
0 & C_{1}^{*}(t) C_{2}(t) & \left|C_{2}(t)\right|^{2} & 0 \\
0 & 0 & 0 & 1-\left|C_{1}(t)\right|^{2}-\left|C_{2}(t)\right|^{2}
\end{array}\right) .
$$

Before proceeding with the Results, we accentuate two important details. First, it is important to stress that since we are considering initial states of the form $|\psi(0)\rangle=C_{01}|10\rangle+$ $C_{02}|01\rangle$, it is possible to parametrize the initial coefficients as $C_{01}=\sqrt{\frac{1-s}{2}}$ and $C_{02}=\sqrt{\frac{1+s}{2}} e^{i \phi}$, where $-1 \leqslant s \leqslant 1$ and $0 \leqslant \phi \leqslant \pi$. Note that in the case where $s=0$, the initial state is entangled, and if $|s|=1$, it is separable, so we refer to the parameter $s$ as the initial separability. Second, it is critical to mention that there exist some very specific initial states. For instance, if $|\psi(0)\rangle=\left(a_{2} / a_{t}\right)|10\rangle-\left(a_{1} / a_{t}\right)|01\rangle$, the state is called subradiant and is a constant solution of Eqs. (18) and (19) that does not decay with time. On the contrary, if $|\psi(0)\rangle=\left(a_{1} / a_{t}\right)|10\rangle+\left(a_{2} / a_{t}\right)|01\rangle$, the state is orthogonal to the previous one, is called superradiant, and is the one that evolves the most rapidly, as shown later.

\section{RESULTS}

\section{A. Quantum metrology: QSL, entanglement, and optimal control}

In the first part of this section, we focus on the potentiality of all initial states to act as metrological probes, in order to then identify our best candidate. To do so, we consider the best metrological candidate as the state that achieves the greatest $\mathcal{F}_{\tilde{\lambda}}^{\text {(tot) }}$ throughout an evolution, where $\tilde{\lambda}$ can be characterizing either the environment, the interaction, or even the quantum probe itself. As stated before, a higher $\mathcal{F}_{\tilde{\lambda}}^{\text {(tot) }}$ implies a greater possibility of achieving a better degree of precision at some particular time. In this framework, a natural question arises: Is there a common feature characterizing the best metrological state for estimating each different subset of parameters? For instance, is entanglement truly useful for improving the estimation or is there a more fundamental physical reason that can be exploited? With this question in mind, in Fig. 1 we study the $\mathcal{F}_{\tilde{\lambda}}^{\text {(tot) }}$ for different classes of parameters, characterizing either the interaction (time $t$ and vacuum Rabi frequency $\mathcal{R}$ ), the environment (width $\lambda$ of the Lorentzian spectral density), or the quantum probe itself (initial phase $\phi$ ). All these quantities are plotted as a function of the initial separability $s$, fixing the interaction parameters at $a_{1}=0.4, a_{2}=0.6, \mathcal{R}=5$, and $T=2$. We point out that the same results were obtained by fixing other interaction parameters and final evolution times.

It is clear in Fig. 1 that the best metrological candidate is the superradiant state since it is the one that maximizes the $\mathcal{F}_{\tilde{\lambda}}^{(\text {tot })}$, for estimating either a parameter of the interaction [Figs. 1(a) and 1(b)], a parameter of the environment [Fig. 1(c)], or even a parameter describing the initial state of the quantum probe itself [Fig. 1(d)]. On the other hand, as can be intuitively deduced from what is expressed in Sec. V, considering that the subradiant state does not decay with time, it is not surprising that this state cannot extract information from the interaction or from the environment. This is the reason why $\mathcal{F}_{\tilde{\lambda}}^{\text {(tot) }}$ is 0 for the situations covered in Figs. 1(a)-1(c). Nevertheless, when a parameter of the initial state of the probe is being estimated, such as the initial phase $\phi$, no interaction with the environment is needed and so $\mathcal{F}_{\phi}^{(\text {tot })}$ is not 0 for this particular initial state, as shown by the asterisk on the dotted blue curve in Fig. 1(d).

In summary, we point out that our best metrological candidate is not the maximally entangled one. This is consistent with the fact that not all entangled quantum states are useful for quantum metrology and they often suffer more from certain nonunitary processes [43-45]. Thus, the question of what is the physical reason underlying the best precision of estimation in our system remains unanswered. As an approach to this problem, we return to what has been pointed out in Sec. III, regarding that there is a relation between the QSL 

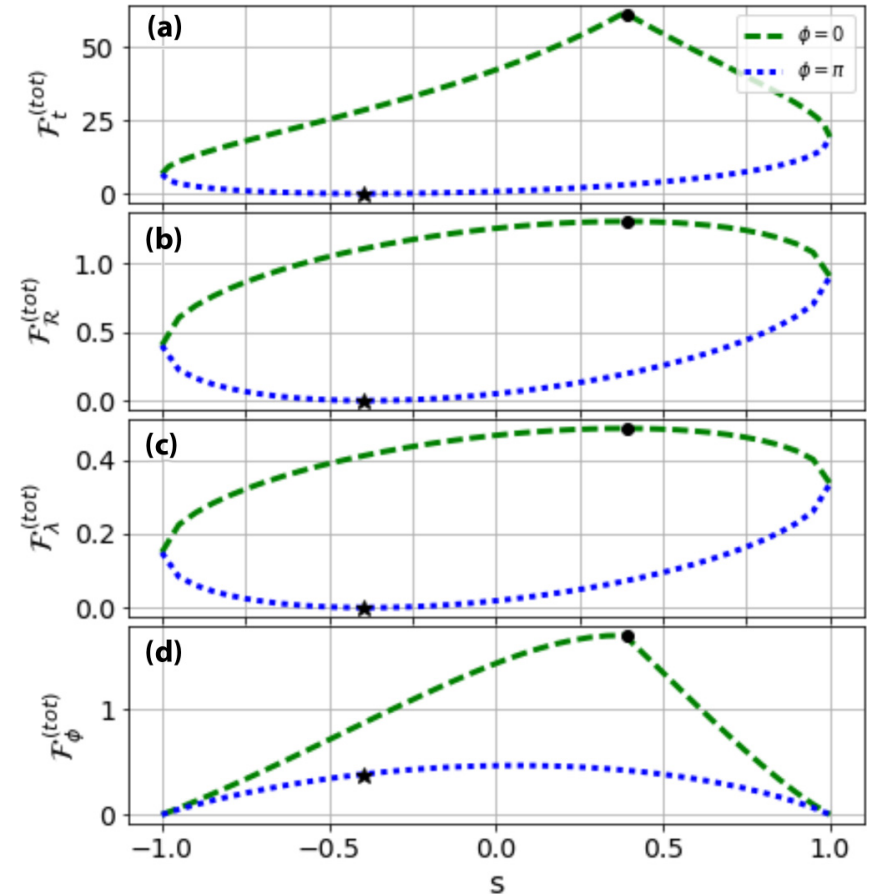

FIG. 1. (a) $\mathcal{F}_{t}^{\text {(tot) }}$ for time estimation; (b) $\mathcal{F}_{\mathcal{R}}^{\text {(tot) }}$ for estimation of the interaction parameter $\mathcal{R}$; (c) $\mathcal{F}_{\lambda}^{(\text {tot })}$ for environment estimation; (d) $\mathcal{F}_{\phi}^{\text {(tot) }}$ for phase estimation. In all panels, the black asterisk corresponds to the subradiant state $\left(s^{*} \simeq-0.38, \phi=\pi\right)$, while the black circle corresponds to the superradiant state $\left(s^{*} \simeq 0.38, \phi=0\right)$. Interaction parameters are fixed at $a_{1}=0.4, a_{2}=0.6, \mathcal{R}=5$, and $T=2\left(\hbar=1\right.$; in units of $\left.1 / \omega_{0}\right)$, while $\lambda$ is set equal to 1 . Any other initial state with a different $\phi$ will reside inside the topological structures found above.

given by Eq. (9) and the total QFI for time estimation $\mathcal{F}_{t}^{(\text {tot })}$. This relation implies that the QFI for time estimation can be interpreted as a measure of the speed of evolution. Therefore, it is obvious that the initial state that maximizes $\mathcal{F}_{t}^{(\text {tot })}$ will at the same time minimize the QSL defined by Eq. (9). However, while this is true for the QFI for time estimation, this is certainly not obvious in cases where other parameters are being estimated, such as $\mathcal{R}, \lambda$, and $\phi$. For example, while the speed of evolution of the subradiant state is 0 for all time, we still have a nonzero $\mathcal{F}_{\phi}^{\text {(tot) }}$ for phase estimation, as shown by the dotted blue curve in Fig. 1(d). For this reason, since these other quantities are not related (in principle) to the speed of evolution, to explore thoroughly the interplay between the accuracy of estimation of different parameters and the speed of evolution seems worthwhile. As a consequence, in Fig. 2 we plot $\tau_{\mathcal{F}}$ but also $\tau_{\mathrm{op}}$, which is a QSL of a different nature (i.e., not based on the QFI), for the same set of parameters as in Fig. 1. We remark that the same results were obtained by fixing different interaction parameters and distances.

The first remarkable thing to note in Fig. 2 is that the two QSLs have a similar behavior, despite their different natures. The two quantities are able to identify both the subradiant and the superradiant states. While the subradiant state takes an infinite time to travel a given predetermined distance since it does not decay with time, the superradiant state is the one that evolves the most rapidly. As can be seen, the QSL given

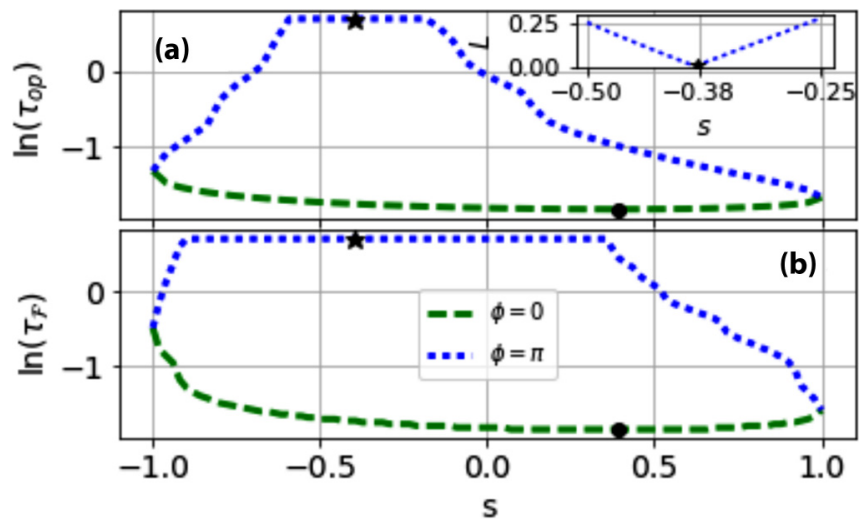

FIG. 2. All quantities are plotted as a function of the initial separability $s$ and for two initial $\phi$ values. (a) $\ln \left(\tau_{\mathrm{op}}\right)$ given by Eq. (10) and fixing $\mathcal{L}=\pi / 4$. Inset: The actual distance $L$ [quantified by the right-hand side of Eq. (10)] traveled by the system within the region near the subradiant state, where the evolution is too slow to reach the predetermined distance given by the geodesic. (b) $\ln \left(\tau_{\mathcal{F}}\right)$ given by Eq. (9) and fixing $\mathcal{L}=\pi / 4$. In both panels, the black asterisk corresponds to the subradiant state $\left(s^{*} \simeq-0.38, \phi=\pi\right)$, while the black circle corresponds to the superradiant state $\left(s^{*} \simeq 0.38, \phi=0\right)$. Parameters are the same as in Fig. 1. The same results were obtained by fixing different interaction parameters and Bures distance $\mathcal{L}$.

by $\tau_{\text {op }}$ proves to be more sensitive for identifying the slow states such as the ones near the subradiant state. In this region, states are so slow that although they are allowed to evolve during a long time, they cannot reach the predetermined distance established by the geodesic, as illustrated in the inset in Fig. 2(a). For this reason, we can use the $\tau_{\mathrm{op}}$ as a tool to explore in more detail the speed of evolution of all possible initial states and then identify more precisely the subradiant state, as shown in Fig. 3.

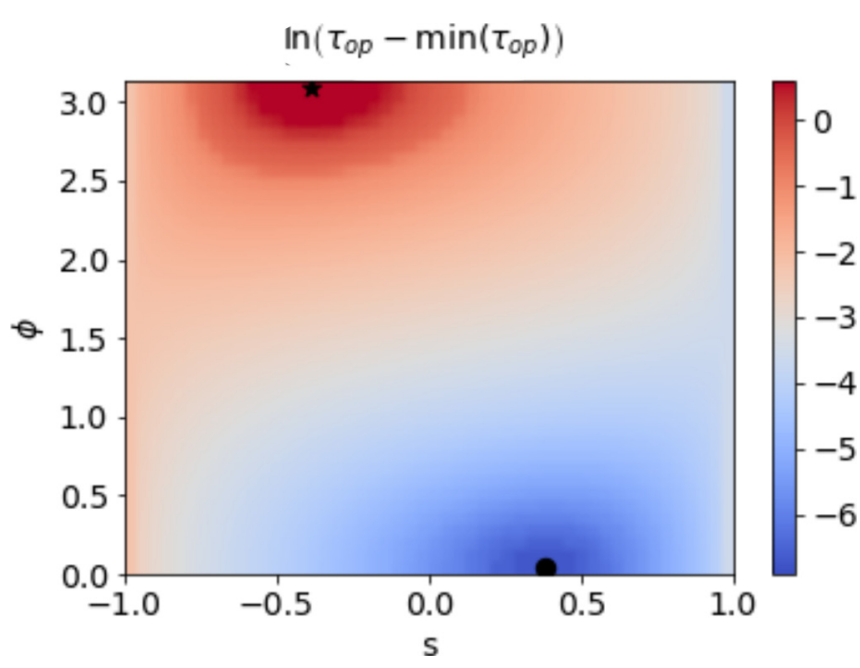

FIG. 3. The $\tau_{\mathrm{op}}$ on a logarithmic scale, having previously subtracted the minimum value from all values so that the regions are well contrasted. The black asterisk corresponds to the subradiant state; the black circle, to the superradiant state. Parameters are the same as in Fig. 1. The same results were obtained by fixing different interaction parameters and Bures distance $\mathcal{L}$. 
As is clear from all the foregoing figures, the best metrological candidate is the superradiant state, since it is not only the fastest but also maximizes the $\mathcal{F}_{\tilde{\lambda}}^{\text {(tot) }}$, for estimating a parameter characterizing either the interaction, the environment, or even the quantum probe itself. However, imagine you are an experimental physicist and you have to measure your observable at a given particular time $T$. Therefore, the fact that $\mathcal{F}_{\tilde{\lambda}}(t)$ shows a dynamical behavior implies that if $\mathcal{F}_{\tilde{\lambda}}^{\text {(tot) }}$ is huge along the whole evolution this will be absolutely useless unless, at the particular time $T, \mathcal{F}_{\tilde{\lambda}}(T)$ reaches its maximum. In this sense, a possible strategy would be to implement some sort of control field so as to maximize the final value $\mathcal{F}_{\tilde{\lambda}}(T)$ and consequently achieve a better degree of precision in the estimation of your parameter $\tilde{\lambda}$ at that particular final time at which the measurement is done [46]. In this context, an interesting question is that the fact that one has a higher $\mathcal{F}_{\tilde{\lambda}}^{\text {(tot) }}$ along a certain total evolution necessarily implies that if one implements an optimization over the final value $\mathcal{F}_{\tilde{\lambda}}(T)$, this final value will be accordingly large. In other words, is the total information flow somehow accommodated by the control field? If this is true, then we should be able to reconstruct the same qualitative topology in some panels in Fig. 1, by plotting the optimal final value $\mathcal{F}_{\tilde{\lambda}}(T)$ obtained by the optimization as a function of the initial separability $s$ and for different $\phi$ values.

In order to study this, we have numerically optimized the coupled differential Eqs. (18) and (19) to find an optimal field $\epsilon(t)$ that maximizes the functional $\mathcal{F}_{\tilde{\lambda}}(T)$. We have resorted to finite-length piecewise constant controls, where the control function $\epsilon(t)$ was taken as a vector of control variables $\epsilon(t) \rightarrow$ $\left\{\epsilon_{k}\right\} \equiv \vec{\epsilon}$, a field with constant amplitude $\epsilon_{k}$ for each time step [47]. The optimization was done by dividing the driving time $T$ into eight equidistant time steps $(k=1,2, \ldots, 8)$, exploring several random initial seeds and using standard optimization tools from the PYTHON SCIPY library [48]. We stress that high-fidelity controls can now be routinely implemented on several different physical systems, such as cold atoms, nitrogen-vacancy centers in diamond, and nuclear magnetic resonance [49-54]. The optimal results obtained by numerical optimization, to estimate both the parameter $\mathcal{R}$ of the interaction and the width $\lambda$ of the Lorentzian spectral density of the environment for a given evolution time $T$, are shown in Fig. 4.

As shown in Fig. 4 and intuitively suggested previously, the fact that we have a certain degree of total QFI $\mathcal{F}_{\tilde{\lambda}}^{\text {(tot) }}$ throughout a whole process (see light dotted-dashed lines in Fig. 4) allows us to manipulate that total flow of information with a control field so as to have a maximum for $\mathcal{F}_{\tilde{\lambda}}(T)$ at some particular fixed evolution time $T$ which is of experimental interest (see dashed lines in Fig. 4). Let us note that this evolution time $T$ is a completely arbitrary time that we had to set for the optimization, but identical results are obtained by setting other evolution times $T$. However, we point out that in realistic experiments the evolution time should not be long enough to avoid the destructive effects generated by decoherence, but also cannot be arbitrarily short considering the fundamental limit imposed by the theory of the QSL [52]. As can be noted, the maximum value achievable with optimal control of $\mathcal{F}_{\tilde{\lambda}}(T)$ depends exclusively on the total degree of information that we had previously within the noncontrolled
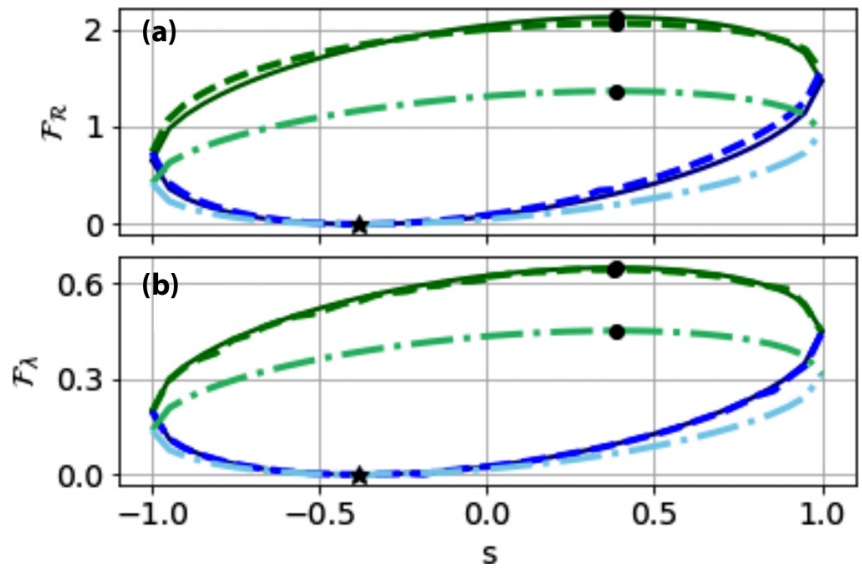

FIG. 4. (a) QFI of the parameter $\mathcal{R}$ characterizing the interaction. (b) QFI of the width $\lambda$ of the spectral density of the environment. In both panels, all green curves correspond to the case with $\phi=0$; blue ones, to $\phi=\pi$. At the same time, the dashed line refers to the optimal final value $\mathcal{F}_{\tilde{\lambda}}(T)$ obtained by the optimization; the dark solid line, to the global maximum of $\mathcal{F}_{\tilde{\lambda}}(t)$ reached throughout the noncontrolled evolution; and, finally, the light dotted-dashed line, to $\mathcal{F}_{\tilde{\lambda}}^{\text {(tot) }}$ [given by Eq. (6)], also throughout the noncontrolled evolution. Interaction parameters are fixed at $a_{1}=0.4, a_{2}=0.6, \mathcal{R}=10$, and $T=2\left(\hbar=1\right.$; in units of $\left.1 / \omega_{0}\right)$. The same results were obtained by fixing other interaction parameters and final evolution times $T$.

scenario. In addition, this maximum value achieved for $\mathcal{F}_{\tilde{\lambda}}(T)$ by optimizing the control field for a specific final time $T$ coincides with the global maximum of $\mathcal{F}_{\tilde{\lambda}}(t)$ reached at an arbitrary time throughout the whole noncontrolled evolution $\left(\mathcal{F}_{\tilde{\lambda}}^{\text {(opt) }}(T)=\max \left(\mathcal{F}_{\tilde{\lambda}}(t)\right) \forall t \in[0, T]\right)$, as is clear upon comparing the dark solid lines with the dashed ones in Fig. 4. Note that this last statement is consistent with the way in which we are controlling the system, i.e., instead of adding an extra interaction term in the Hamiltonian we are controlling it by tuning its frequency with finite-length piecewise constant controls, as we discuss following Eq. (14). Therefore, even though the optimization itself is a complicated method of finding optimal solutions for the maximization of a given functional, it makes sense that exploring the multidimensional control landscape in frequency space addresses the system towards the global maximum that was reached at an arbitrary time during the noncontrolled dynamics for any of the parameters of interest $[47,55]$. This is a numerical sample of the information flows being actively manipulated by the control field, a statement that is explored in more detail in the next subsection.

\section{B. Exploiting information flows for quantum control}

An interesting point to note is based on the fact that the concept of information flow may be applied to an entire set of different physical quantities, for instance, QFI, NM, and also entanglement. So a natural question can be formulated: How are these information flows related to each other? In order to address this question, we use the control method presented previously as a way to dive through the subspace of the best solutions and try to extract unknown relations from them. For simplicity, we focus our attention just on the incoming 


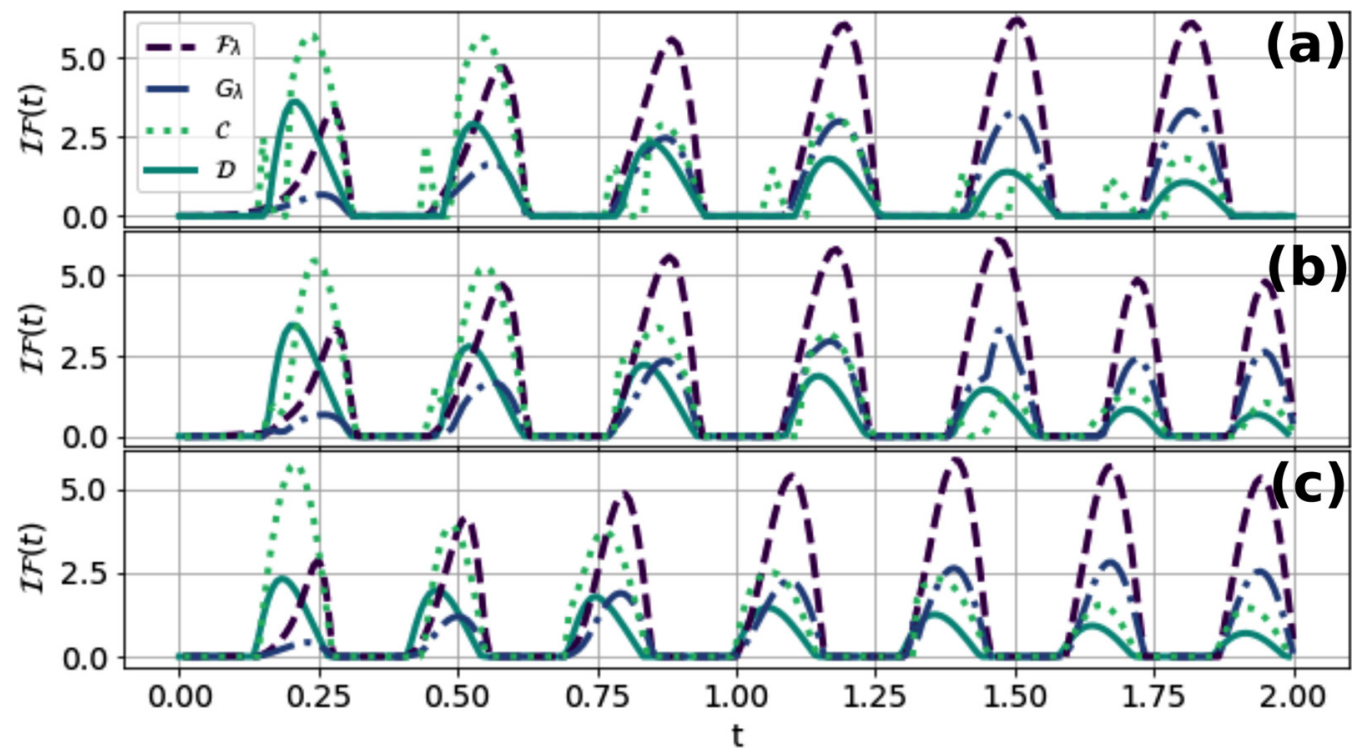

FIG. 5. Incoming flows for $\mathcal{F}_{\lambda}(t), G_{\lambda}(t), \mathcal{D}(t)$, and $\mathcal{C}(t)$ as a function of time for the following set of situations: (a) no control; (b) with control maximizing $\mathcal{F}_{\lambda}(T)$; and (c) with control maximizing $\mathcal{C}(T)$. Interaction parameters are fixed at $a_{1}=0.4, a_{2}=0.6, \mathcal{R}=10$, and $T=2$ ( $\hbar=1$; in units of $\left.1 / \omega_{0}\right)$, while $\lambda$ is set equal to 1 and the initial state is maximally entangled $(s=0$ and $\phi=0)$. The POVM used for computing $G_{\lambda}$ from Eq. (4) was $P_{x}=\left\{E_{1}, E_{2}, E_{3}\right\}$, where $E_{1}=\sqrt{2} /(1+\sqrt{2})|10\rangle\left\langle 10\left|, E_{2}=\sqrt{2} /(1+\sqrt{2})\right| 01\right\rangle\langle 01|$, and $E_{3}=\mathbb{1}-E_{1}-E_{2}$. Analogous results were obtained by fixing different interaction parameters and final evolution times $T$.

flows, i.e., the time intervals in which these quantities are an increasing function of time, and analyze whether or not they are related in different controlled situations. With this purpose, we define the incoming flow $\mathcal{I} \mathcal{F}(t)$ as

$$
\mathcal{I F}(t)=\frac{d A(t)}{d t}>0
$$

where $A(t)=\left\{\mathcal{F}_{\tilde{\lambda}}(t), G_{\tilde{\lambda}}(t), \mathcal{D}(t), \mathcal{C}(t)\right\}, \mathcal{F}_{\tilde{\lambda}}(t)$ being the QFI for some specific parameter $\tilde{\lambda}, G_{\tilde{\lambda}}(t)$ the Fisher information for a given POVM estimating the same specific parameter $\tilde{\lambda}$, $\mathcal{D}(t)$ the distinguishability between the two initial states that maximize the BLP measure in Eq. (12), and, finally, $\mathcal{C}(t)$ the concurrence between both atoms. Let us remark that all the above quantities are time dependent and it is their dynamics that we intend to relate. As an illustrative and representative example, in Fig. 5 we plot all these incoming flows as a function of time for the case of environment estimation $(\tilde{\lambda}=\lambda)$ and under the following set of different situations: without any control field [Fig. 5(a)], with a control field that maximizes $\mathcal{F}_{\lambda}(T)$ [Fig. 5(b)], and, finally, for a control protocol that maximally preserves $\mathcal{C}(T)$ [Fig. 5(c)], where $T$ is a completely arbitrary final evolution time that is fixed.

Something we can especially note in all the panels in Fig. 5 is based on the fact that there is a univocal dynamical relation between $\mathcal{F}_{\lambda}(t)$ for estimating a parameter of the environment and the revivals of distinguishability $\mathcal{D}(t)$, which are a common feature of NM and normally interpreted as a backflow of information that flows from the environment to the reduced open system. In this way, one could intuitively think that initially there is no information about the unknown parameter of the spectral density since, to extract information about it, an interaction with the degrees of freedom of the environment must occur. But surprisingly, this interaction seems not to be a sufficient requirement unless we are in a time interval in which we are experiencing a backflow of information, something that may only occur in a non-Markovian quantum evolution. It is clear in all the panels in Fig. 5 that the only intervals in which we are gaining information about the environment are those during which a backflow of information is occurring. Whenever the backflow stops and therefore we start to lose information, $\mathcal{F}_{\lambda}(t)$ decreases.

In a similar way, as shown in Fig. 6, the same dynamical relation between the QFI and NM arises when the estimation is for a parameter that characterizes the probe itself, such as its initial phase $\phi$. In this case, as the system starts to interact with the environment the information about the initial

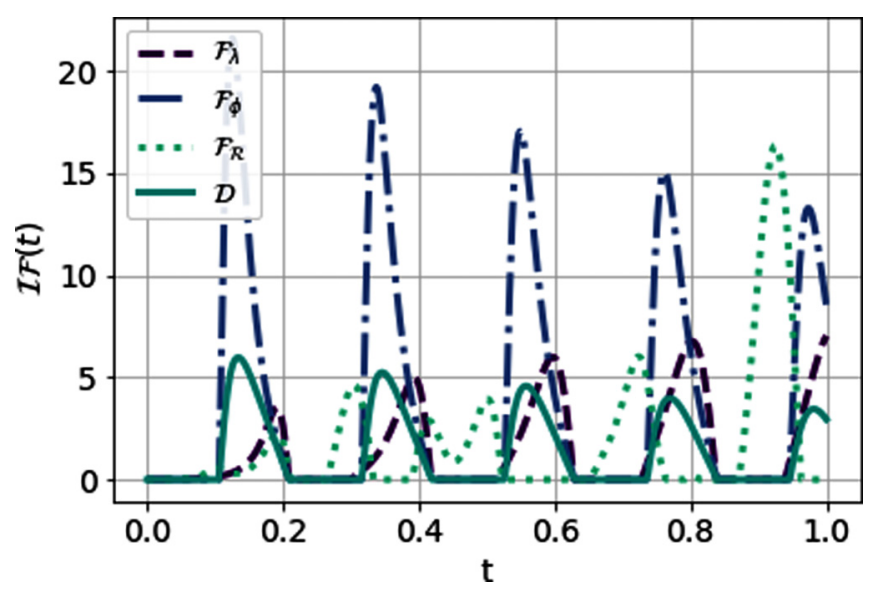

FIG. 6. Incoming flows for $\mathcal{F}_{\mathcal{R}}(t), \mathcal{F}_{\phi}(t), \mathcal{F}_{\lambda}(t)$, and $\mathcal{D}(t)$ as a function of time for the noncontrolled situation. Interaction parameters are fixed at $a_{1}=0.25, a_{2}=0.75, \mathcal{R}=15$, and $T=1(\hbar=1$; in units of $\left.1 / \omega_{0}\right)$, while $\lambda$ is set equal to 1 and the initial state is maximally entangled ( $s=0$ and $\phi=0$ ). The same results were obtained by fixing different interaction parameters, initial states, and final evolution times $T$. 
phase is leaked into it and is only due to the revivals of distinguishability and the backflow of information that the QFI of this initial phase increases again. On the contrary, it is not surprising that in a situation where the estimation is for a parameter characterizing the interaction (such as $\mathcal{R}$ ), no backflow of information is needed and thus the QFI can increase independently of being in a non-Markovian time interval, as can be appreciated from the dotted green line in Fig. 6. Here the probe just needs to interact (somehow) with the environment so as to extract information about the interaction, without the necessity of receiving any backflow of information.

In summary, considering that QFI has a clear practical meaning and that there is no discussion with respect to whether or not it is useful for specific tasks (i.e., for quantum metrology), the result linking QFI and NM in this specific model gives the latter a clear and indisputable meaning as a quantum resource, either to estimate a parameter of the environment or even to maximally preserve the information on a parameter characterizing the reduced open system $[25,26,56-59] .{ }^{1}$ We hope this numerical finding to motivate further theoretical as well as experimental investigations.

Let us return now to Fig. 5 and focus on the controlled scenarios that are being studied. For instance, Fig. 5(b) shows the behavior of all the incoming flows when the functional $\mathcal{F}_{\lambda}(T)$ is being maximized. The main difference from the noncontrolled scenario presented in Fig. 5(a) is that now the information flows corresponding both to QFI and to NM are accommodated by the control in order to exhibit a maximum value for $\mathcal{F}_{\lambda}(T)$ at the end of the protocol. Indeed, while in Fig. 5(a) the last revival of both $\mathcal{D}(t)$ and $\mathcal{F}_{\lambda}(t)$ ends at a time $t<T$, in Fig. 5(b) we can see that the control has accommodated the dynamics so as to make the last revival end at a time $t \simeq T$ and thus achieve a maximum for the QFI at that final evolution time $T$.

Finally, with regard to entanglement and its interplay between QFI and NM, in the scenarios covered in Figs. 5(a) and 5(b), it is clear that there is no relation at all between these three quantities. For instance, what Fig. 5(a) shows us is that the concurrence $\mathcal{C}(t)$ between the atoms may increase independently of being in a time interval in which a backflow of information is being manifested. This is not surprising since it is well known that a common Markovian environment may induce some degree of noncontrolled entanglement between two noninteracting parts coupled to it $[25,60,61]$. With respect to Fig. 5(b), where the control task is to optimize the final value of $\mathcal{F}_{\lambda}(T)$, as shown in Figs. 1 and 4, entanglement is not the crucial factor for enhancing the parameter estimation. So it is not surprising not to have a clear correlation in this particular situation. However, the most interesting point to stress arises in Fig. 5(c), where the control task now consists in the preservation of entanglement at time $T$. In this case, all the quantities are clearly correlated. The best way that

\footnotetext{
${ }^{1} \mathrm{~A}$ minor comment, but important to highlight, is that considering that the QFI is defined from a maximization over all possible measurements, it is crucial to show that there exists at least one particular POVM that exhibits the same dynamical behavior. This is shown for $G_{\lambda}$ in all panels in Fig. 5.
}

the control field finds to preserve entanglement is to take advantage whenever a backflow of information is occurring, so as to recover from this backflow the entanglement that was previously washed out when the information was being lost to the environment.

\section{FINAL REMARKS}

In this work, we have studied the problem of quantum metrology in the framework of an open quantum system subject to a non-Markovian quantum evolution. The main motivation was to deepen our understanding of the relationship between apparently disconnected physical quantities, such as QFI, the QSL, NM, and entanglement. By addressing this complicated problem in a particular but fully analytical and controlled physical system, we have first shown that the speed of evolution and the accuracy of estimation are deeply connected. In this way, upon exploring the process of estimation for a variety of different classes of parameters, the best metrological candidate proved to be the state that evolved the most rapidly, and not the maximally entangled one. This was shown by the use of the QSL.

Another important key point of our work is based on the possibility of controlling externally the composite quantum system used as a probe. In this context, by maximizing the value of the QFI at a given final evolution time, we have shown how the total QFI flow throughout the evolution can be exploited and accommodated by the control field in order to achieve the best precision of estimation at that final evolution time, which may be of experimental interest. This could certainly be useful in current experiments [20].

Finally, by using the optimal control method not as an end in itself, but as a tool to explore the best solutions and extract unnoticed relations from them $[25,26]$, we have focused on the dynamics of QFI, NM, and entanglement to determine whether or not these quantities are correlated under different control schemes. In all the scenarios considered, we have found a direct dynamical relation between the incoming flows of QFI (for both environment and phase estimation) and the revivals of distinguishability, which gives NM a concrete use as a resource for quantum metrology. With regard to entanglement and its dynamical interplay with QFI and NM, we have shown that when we optimize a control field to maximally preserve the initial entanglement at a fixed final evolution time, the incoming flows of entanglement coincide perfectly with the incoming flows of QFI and distinguishability. In other words, as information is being recovered from the environment, via the revivals of QFI and distinguishability, this backflow is used here by the control to retrieve the entanglement that was previously washed out when the information was being leaked into it.

With the results obtained, we sincerely expect this work to shed light and clarity on the problem of quantum metrology and its deep connection with QSL, NM, and entanglement.

\section{ACKNOWLEDGMENTS}

This work was partially supported by CONICET (PIP $11220150100493 \mathrm{CO}), \mathrm{UBACyT}$ (20020130100406BA), ANPCyT (PICT-2016-1056), and the National Science Foundation (Grant No. PHY-1630114). 
[1] V. Giovannetti, S. Lloyd, and L. Maccone, Nat. Photon. 5, 222 (2011).

[2] C. L. Degen, F. Reinhard, and P. Cappellaro, Rev. Mod. Phys. 89, 035002 (2017).

[3] J. J. Bollinger, W. M. Itano, D. J. Wineland, and D. J. Heinzen, Phys. Rev. A 54, R4649 (1996).

[4] S. F. Huelga, C. Macchiavello, T. Pellizzari, A. K. Ekert, M. B. Plenio, and J. I. Cirac, Phys. Rev. Lett. 79, 3865 (1997).

[5] V. Giovannetti, S. Lloyd, and L. Maccone, Science 306, 1330 (2004).

[6] V. Giovannetti, S. Lloyd, and L. Maccone, Phys. Rev. Lett. 96, 010401 (2006).

[7] J. Esteve, C. Gross, A. Weller, S. Giovanazzi, and M. Oberthaler, Nature 455, 1216 (2008).

[8] M. G. Paris, Int. J. Quantum. Info. 7, 125 (2009).

[9] U. Dorner, R. Demkowicz-Dobrzanski, B. J. Smith, J. S. Lundeen, W. Wasilewski, K. Banaszek, and I. A. Walmsley, Phys. Rev. Lett. 102, 040403 (2009).

[10] Y. Watanabe, T. Sagawa, and M. Ueda, Phys. Rev. Lett. 104, 020401 (2010).

[11] X.-M. Lu, X. Wang, and C. P. Sun, Phys. Rev. A 82, 042103 (2010).

[12] J. Ma, Y. X. Huang, X. Wang, and C. P. Sun, Phys. Rev. A 84, 022302 (2011).

[13] A. W. Chin, S. F. Huelga, and M. B. Plenio, Phys. Rev. Lett. 109, 233601 (2012).

[14] W. Zhong, Z. Sun, J. Ma, X. Wang, and F. Nori, Phys. Rev. A 87, 022337 (2013).

[15] S. Alipour, M. Mehboudi, and A. T. Rezakhani, Phys. Rev. Lett. 112, 120405 (2014).

[16] Y.-L. Li, X. Xiao, and Y. Yao, Phys. Rev. A 91, 052105 (2015).

[17] C. Benedetti, F. Salari Sehdaran, M. H. Zandi, and M. G. A. Paris, Phys. Rev. A 97, 012126 (2018).

[18] H. R. Jahromi, M. Amini, and M. Ghanaatian, Quant. Info. Proc. 18, 338 (2019).

[19] F. S. Sehdaran, M. H. Zandi, and A. Bahrampour, Phys. Lett. A 383, 126006 (2019)

[20] S. Xu, H. Z. Shen, X. X. Yi, and W. Wang, Phys. Rev. A 100, 032108 (2019).

[21] G. L. Giorgi, S. Longhi, A. Cabot, and R. Zambrini, Ann. Phys. 531, 1900307 (2019).

[22] F. Gebbia, C. Benedetti, F. Benatti, R. Floreanini, M. Bina, and M. G. A. Paris, Phys. Rev. A 101, 032112 (2020).

[23] H.-P. Breuer, E.-M. Laine, and J. Piilo, Phys. Rev. Lett. 103, 210401 (2009).

[24] A. Rivas, S. F. Huelga, and M. B. Plenio, Rep. Prog. Phys. 77, 094001 (2014).

[25] N. Mirkin, P. Poggi, and D. Wisniacki, Phys. Rev. A 99, 062327 (2019).

[26] N. Mirkin, P. Poggi, and D. Wisniacki, Phys. Rev. A 99, 020301(R) (2019).

[27] F. Verstraete, M. M. Wolf, and J. I. Cirac, Nat. Phys. 5, 633 (2009).

[28] C.-F. Li, G.-C. Guo, and J. Piilo, Europhys. Lett. 128, 30001 (2020).

[29] S. Deffner and E. Lutz, Phys. Rev. Lett. 111, 010402 (2013).

[30] A. Del Campo, I. L. Egusquiza, M. B. Plenio, and S. F. Huelga, Phys. Rev. Lett. 110, 050403 (2013).

[31] M. M. Taddei, B. M. Escher, L. Davidovich, and R. L. de Matos Filho, Phys. Rev. Lett. 110, 050402 (2013).
[32] N. Mirkin, F. Toscano, and D. A. Wisniacki, Phys. Rev. A 94, 052125 (2016).

[33] N. Mirkin, F. Toscano, and D. A. Wisniacki, Phys. Rev. A 97, 046101 (2018).

[34] B. Escher, R. de Matos Filho, and L. Davidovich, Nat. Phys. 7, 406 (2011)

[35] G. Salvatori, A. Mandarino, and M. G. A. Paris, Phys. Rev. A 90, 022111 (2014).

[36] C. W. Helstrom, Quantum Detection and Estimation Theory (Academic Press, New York, 1976).

[37] A. S. Holevo, Probabilistic and Statistical Aspects of Quantum Theory, Vol. 1 (Springer Science \& Business Media, New York, 2011).

[38] L. Mandelstam and I. Tamm, Izv. Akad. Nauk SSSR 9, 122 (1945).

[39] N. Margolus and L. B. Levitin, Physica D: Nonlin. Phenom. 120, 188 (1998).

[40] L. B. Levitin and T. Toffoli, Phys. Rev. Lett. 103, 160502 (2009).

[41] S. Maniscalco, F. Francica, R. L. Zaffino, N. Lo Gullo, and F. Plastina, Phys. Rev. Lett. 100, 090503 (2008).

[42] R. L. Franco, B. Bellomo, S. Maniscalco, and G. Compagno, Int. J. Mod. Phys. B 27, 1345053 (2013).

[43] P. Hyllus, O. Gühne, and A. Smerzi, Phys. Rev. A 82, 012337 (2010).

[44] F. Fröwis, M. Skotiniotis, B. Kraus, and W. Dür, New J. Phys. 16, 083010 (2014).

[45] S. Altenburg, S. Wölk, G. Tóth, and O. Gühne, Phys. Rev. A 94, 052306 (2016).

[46] J. Liu and H. Yuan, Phys. Rev. A 96, 012117 (2017).

[47] M. Larocca, E. Calzetta, and D. A. Wisniacki, Phys. Rev. A 101, 023410 (2020).

[48] E. Jones, T. Oliphant, and P. Peterson, SciPy: Open source scientific tools for Python (2001), http://www.scipy.org.

[49] X. Rong, J. Geng, Z. Wang, Q. Zhang, C. Ju, F. Shi, C.-K. Duan, and J. Du, Phys. Rev. Lett. 112, 050503 (2014).

[50] F. Dolde, V. Bergholm, Y. Wang, I. Jakobi, B. Naydenov, S. Pezzagna, J. Meijer, F. Jelezko, P. Neumann, T. SchulteHerbrüggen et al., Nat. Commun. 5, 3371 (2014).

[51] J. Scheuer, X. Kong, R. S. Said, J. Chen, A. Kurz, L. Marseglia, J. Du, P. R. Hemmer, S. Montangero, T. Calarco et al., New J. Phys. 16, 093022 (2014).

[52] S. van Frank, M. Bonneau, J. Schmiedmayer, S. Hild, C. Gross, M. Cheneau, I. Bloch, T. Pichler, A. Negretti, T. Calarco et al., Sci. Rep. 6, 34187 (2016).

[53] J. P. Peterson, H. Katiyar, and R. Laflamme, arXiv:2006.10133.

[54] A. Larrouy, S. Patsch, R. Richaud, J.-M. Raimond, M. Brune, C. P. Koch, and S. Gleyzes, Phys. Rev. X 10, 021058 (2020).

[55] H. A. Rabitz, M. M. Hsieh, and C. M. Rosenthal, Science 303, 1998 (2004).

[56] B. Bylicka, D. Chruściński, and S. Maniscalco, arXiv:1301.2585.

[57] D. M. Reich, N. Katz, and C. P. Koch, Sci. Rep. 5, 12430 (2015).

[58] N. Anand and T. A. Brun, arXiv:1903.03880.

[59] G. D. Berk, A. J. Garner, B. Yadin, K. Modi, and F. A. Pollock, arXiv:1907.07003.

[60] F. Benatti, R. Floreanini, and M. Piani, Phys. Rev. Lett. 91, 070402 (2003).

[61] B. Kraus, H. P. Büchler, S. Diehl, A. Kantian, A. Micheli, and P. Zoller, Phys. Rev. A 78, 042307 (2008). 